\title{
Analytical validation of an mRNA-based urine test to predict the presence of high-grade prostate cancer
}

Daphne Hessels ${ }^{1}$, Hans de Jong ${ }^{1}$, Sander A. Jannink', Michael Carter ${ }^{2}$, Manuel Krispin², Wim Van Criekinge ${ }^{3}$, Leander Van Neste ${ }^{4}$ and Jack A. Schalken ${ }^{5^{*}}$

\begin{abstract}
Background: The challenge for prostate cancer ( $\mathrm{PCa}$ ) diagnosis is to improve the ability to distinguish indolent from aggressive PCa. Increased urinary levels of Distal-less Homeobox 1 (DLX1) and Homeobox C6 (HOXC6) mRNA have been associated with high-grade PCa (Gleason Score $\geq 7$ ). Reverse transcriptase PCR (RT-PCR)-assays for the quantification of DLX1 and HOXC6 mRNA levels were developed, and clinical validation showed that these urinary markers can significantly improve accuracy for detection of high-grade PCa at biopsy. The aim of this study was to validate the analytical performance of these mRNA-based assays, including specimen stability and analytical sensitivity, specificity, precision, repeatability and reproducibility.
\end{abstract}

Methods: Analytical validation of the RT-PCR assays for DLX1, HOXC6 and KLK3 was performed using in vitro transcribed (IVT) specimens of the target genes, covering all aspects of the analytical method including assay sensitivity, specificity, linearity, precision, repeatability and reproducibility using pre-specified acceptance criteria. To confirm that the mRNA assays are sufficient robust for use in clinical routine laboratories, the performance characteristics were verified in an independent laboratory using post-DRE collected urine samples from 101 men scheduled for prostate biopsy.

Results: A stabilization buffer, developed for urine preservation under standard pre-processing conditions, makes sample shipment from clinics to laboratories easily feasible. The mRNA in urine samples, preserved in this stabilization buffer, is stable at room temperature up to 5 days from collection, resulting in $100 \%$ evaluable rate. The long term stability of the mRNAs in the buffer was shown by similar clinical performances using RNA values obtained immediately after urine collection (area under curve (AUC) $0.72(95 \%$ Cl: $0.61-0.83)$ ) and after 1-year storage (AUC 0.71 (95\% Cl: $0.60-0.81)$ ). Test performance was not compromised by most common inhibitors and bacterial strains found in urine. However, an inhibitory effect of hemoglobin was observed.

All precision, reproducibility, instrument and inter-laboratory variation data obtained for the mRNA-based assays met the pre-specified acceptance criteria of a standard deviation less than or equal to 0.5 crossing point. The analysis of 99 whole urine samples at two laboratories indicated a very strong positive correlation $(r=0.997, P<0.001)$. The test outcome in terms of absolute difference in likelihood for high-grade PCa upon biopsy was less than 2\% between the two sites.

Conclusions: This study illustrates the robustness of the mRNA assays, enabling testing in clinical routine laboratories and molecular pathology laboratories where the here described automated RNA extraction and PCR platforms are available.

Keywords: Prostate cancer, Urine, Analytical validation, Biomarkers, RT-PCR

\footnotetext{
* Correspondence: Jack.Schalken@radboudumc.nl

${ }^{5}$ Department of Urology, Radboud University Nijmegen Medical Centre,

Nijmegen, The Netherlands

Full list of author information is available at the end of the article
} 


\section{Background}

In the United States and Europe, prostate cancer (PCa) is one of the most common cancers and one of the leading causes of cancer-related deaths [1, 2]. Globally, in 2012, an estimated 1.1 million men were newly diagnosed with PCa and 307.000 men died from the disease [3]. Autopsy on men aged 80 years and older, who died from other causes, revealed that almost $70 \%$ had $\mathrm{PCa}$ at time of death [4]. This implies that the majority of PCa's are clinically insignificant and will have an indolent disease course. Less than $3 \%$ of men diagnosed with $\mathrm{PCa}$ develop invasive, lethal disease [3]. Since the early 1990's, serum prostate-specific antigen (PSA) testing is widely used to screen for PCa. However, the limited specificity of PSA has resulted in a large number of negative biopsies in healthy males and the diagnosis of indolent cancers, leading to anxiety for potentially aggressive occult PCa and overtreatment [5]. Therefore, the most important challenge is to improve the ability to specifically identify aggressive PCa at the time of diagnosis and in an early stage of the disease.

Novel biomarkers with high specificity for aggressive disease could aid in the decision for prostate biopsy and hereby help to avoid the problem of unnecessary biopsies, overdiagnosis and overtreatment. Previously, a stepwise selection process of mRNA-profiling data was described, in which an mRNA-panel was identified for predicting the presence of high-grade (Gleason score (GS) 27 ) PCa upon biopsy using urinary sediments collected after digital rectal examination (DRE) [6]. The mRNA panel includes the genes Distal-less Homeobox 1 (DLX1) and Homeobox C6 (HOXC6). DLX1 is involved in the acquisition of epithelial-neuroendocrine differentiation, a characteristic associated with aggressive cancer $[7,8]$. HOXC6 is an upregulated homeobox gene in primary, metastasized and castration-resistant PCa [9]. These biomarkers form the basis for a urine test that allows the stratification of patients with high-grade disease. However, analytical validation is key for the successful introduction of these biomarkers to clinical practice.

Because of the ease of collection, and the fact that prostate cells are directly released into the urethra through prostatic ducts after DRE, whole urine has become the future for non-invasive biomarker testing. It is a preferable substrate since it does not require labor-intensive, timeconsuming urine processing procedures, whereas mRNA yield is not compromised avoiding that differences in handling of urine can affect the outcome of the analytical assay. To minimize the variability, the post-DRE urine collection procedure was standardized and the sample preprocessing protocol was optimized with a stabilization buffer specifically developed to this end.

For clinical validation of the DLX1 and HOXC6 biomarkers, real-time polymerase chain reaction (RT-PCR) assays were developed specifically designed for analysis of whole urine samples from men considered for prostate biopsy. The RT-PCR assays were developed to quantify DLX1 and HOXC6 mRNA, in addition to Kallikrein-3 (KLK3) mRNA for reference purposes. The normalization to $K L K 3$ mRNA is used to control analytical variability as well as prostate-related RNA input variability. An RNA value was calculated by summing the normalized ratios of DLX1 and HOXC6 mRNA. In whole urine the RNA value was shown to be a good predictor for the detection of high-grade $\mathrm{PCa}$ upon biopsy $[6,10]$. The robust automated RNA extraction and PCR platforms that form the base of these assays have contributed to the success of these clinical validation studies. The inter- and intralaboratory variability was minimized through the implementation of standardized techniques which were relied upon for both extraction and molecular analysis procedures [11].

Here the analytical validation of the RT-PCR assays for $D L X 1, H O X C 6$ and KLK3 is described, covering all aspects of the analytical method including assay sensitivity, specificity, linearity, precision, repeatability and reproducibility using pre-specified acceptance criteria [12]. To confirm that the mRNA assays are sufficient robust for routine use in diagnostic laboratories, the performance characteristics were verified in an independent Clinical Laboratory Improvement Amendments (CLIA) accredited laboratory.

\section{Methods}

\section{Clinical sample collection}

Voided urine samples were collected from a cohort of 99 men scheduled for prostate biopsies due to elevated PSA levels, an abnormal digital rectal exam (DRE) or a family history of PCa. Subjects were enrolled from six urological outpatient clinics in the Netherlands between July 2014 and December 2014. Men with verified PCa, with prior $\mathrm{PCa}$ diagnosis, undergoing medical therapy known to affect PSA levels, or men that underwent prostate biopsy within 3 months prior to enrolment, or invasive treatment for benign prostatic hyperplasia $(\mathrm{BPH})$ within 6 months prior to enrolment were excluded from the study. All participants signed a consent form that was approved by the institutional review board of the participating clinics. In Table 1, the characteristics of the study population are presented.

After a six-stroke DRE, $30 \mathrm{ml}$ of first void urine was collected and $8 \mathrm{ml}$ was immediately transferred to a urine specimen transport tube containing $2 \mathrm{ml}$ stabilization buffer (Catalogue number UTXL32SE, MDxHealth B.V., Nijmegen, the Netherlands). Samples were shipped at room temperature to MDxHealth clinical diagnostic laboratory (Nijmegen, the Netherlands) on the day of collection, after which the samples were stored at $-80{ }^{\circ} \mathrm{C}$. One 
Table 1 Characteristics of the study population

\begin{tabular}{ll}
\hline & Study cohort $(n=99)$ \\
\hline Age (years) (mean (median:IQR)) & $65.9(66: 62.0-69.0)$ \\
PSA (ng/mL) (mean (median:IQR)) & $46.1(7.4: 5.5-11.6)^{\mathrm{a}}$ \\
Family history of PCa (no-yes-NA) & $43.4 \%-13.1 \%-42.4 \%$ \\
First biopsy ( $\mathrm{n}(\%))$ & $91(92.0 \%)$ \\
Prostate volume (mean (median:IQR)) & $55.5(49: 36-72)$ \\
PSA density (ng/mL x cc-1) (mean & $0.89(0.15: 0.09-0.27)$ \\
(median : IQR)) & $54(54.5 \%)$ \\
DRE (abnormal) (\%) & $54(54.5 \%)$ \\
PCa diagnosis (n (\%)) & $17(31.5 \%)$ \\
$\quad$ Gleason score $\leq 6(n(\%))$ & $18(33.3 \%)$ \\
Gleason score 7 (n (\%)) & $19(35.2 \%)$ \\
$\quad$ Gleason score 8-10 (n (\%))
\end{tabular}

$D R E$ digital rectal examination, $I Q R$ inter quartile range, $N A$ not applicable, $P C a$ prostate cancer, PSA prostate-specific antigen

${ }^{a}$ Four men had serum PSA values of more than $100 \mathrm{ng} / \mathrm{mL}$. Without these outliers the average PSA value was $12.1 \mathrm{ng} / \mathrm{mL}$

$\mathrm{ml}$ of each specimen was used for RT-PCR analysis of the biomarkers in February 2015, and the remainder of the sample was stored at $-80{ }^{\circ} \mathrm{C}$. In December 2015, each specimen was thawed and distributed into 4 aliquots of 1.1 $\mathrm{ml}$, which were stored at $-80{ }^{\circ} \mathrm{C}$. Two aliquots of each sample were shipped on dry ice to an independent CLIA laboratory (MDxHealth Inc., Irvine, California, USA). Throughout this manuscript, the laboratories in Nijmegen and Irvine will be referred to as Lab A and Lab B, respectively.

\section{RNA extraction}

Prior to extraction, the samples were thawed at $37^{\circ} \mathrm{C}$ for $15 \mathrm{~min}$, during which the tubes were inverted every three minutes. Automated nucleic acid isolation using the MagNA Pure 96 Instrument (Roche Life Science, Indianapolis, IN, USA) was performed with $1 \mathrm{ml}$ of whole urine per analysis using the DNA and Viral NA large volume kit (Roche Life Science, Indianapolis, IN, USA) according to the manufacturer's instructions. One $\mathrm{ml}$ of nuclease-free water was used as negative nucleic acid isolation control (NAI-). The RNA of each extracted specimen as well as the NAI- control was eluted in $50 \mu \mathrm{l}$ Elution buffer (Roche Life Science, Indianapolis, IN, USA), the elution plate was sealed and stored in a refrigerator at 2 to $8{ }^{\circ} \mathrm{C}$.

\section{Quantification of DLX1, HOXC6 and KLK3}

For the analytical validation, a prototype amplification kit (Labo Biomedical Products B.V., Rijswijk, the Netherlands) was used. The reagents for the test include a singleplex Taqman $^{\circ}$ Fast virus one-step PCR mastermix (ThermoFisher Scientific, Waltham, MA, USA) for each of the 3 targets, a calibrator (Cal) and a reverse transcriptase (RT)- control. The singleplex mastermixes contain TaqMan ${ }^{\circ}$ probe and primers for specific detection of either $D L X 1$, HOXC6 or KLK3, reverse transcriptase, Taq polymerase, dNTPs and buffer. The calibrator consists of a multi-target plasmid containing the relevant DLX1, HOXC6 and KLK3 sequences. The RT-control is a mix of in vitro transcribed (IVT) RNA of HOXC6, DLX1, and KLK3 target sequences. The calibrator $\left(\mathrm{Cal} 10^{4}\right)$, quantified to 10,000 copies of the multi-target plasmid, and RT-control, quantified to 10,000 copies of IVT RNAs, were used as positive reference controls in each run. Additionally, a NAI- and a notemplate control (NTC) were included in each run. $K L K 3$ was included as an endogenous positive nucleic acid isolation control.

Per RT-PCR reaction, $14 \mu \mathrm{l}$ of RNA specimen or control was mixed with $6 \mu \mathrm{l}$ mastermix in a single well of a LightCycler 480 II multiwell plate (Roche Life Science, Indianapolis, IN, USA). At Lab A, a LightCycler 480 II 96-well plate format was used whereas at Lab B a LightCycler 480 II 384-well plate format was used. Regardless of the plate format, $20 \mu \mathrm{l}$ reaction was reverse-transcribed and amplified in a LightCycler 480 II system (Roche Life Science, Indianapolis, IN, USA) using the following cycling conditions: $10 \mathrm{~min} 50^{\circ} \mathrm{C}$ and $30 \mathrm{~s} 95^{\circ} \mathrm{C}$, followed by 50 cycles each consisting of $5 \mathrm{~s} 95^{\circ} \mathrm{C}$ and $30 \mathrm{~s} 60^{\circ} \mathrm{C}$.

The levels of gene expression were calculated with the absolute quantification analysis using the Crossing point (Cp) as quantification point and the second derivative maximum method implemented by the LightCycler ${ }^{\oplus} 480$ II software version 1.5.0 (Roche Life Science, Indianapolis, IN, USA). The normalized ratio (NR) of the target gene, either HOXC6 or $D L X 1$, relative to the $K L K 3$ reference gene, was calculated using the delta delta $\mathrm{Cp}$ method $(\Delta \Delta \mathrm{Cp})$ [13].

An RNA value was calculated by summing the NR of HOXC6 and DLX1. In the clinical setting, the RNA value is combined with traditional clinical risk factors, including PSA, DRE, prostate volume, age and family history using a logistic regression model described by Van Neste et al. [10]. The output of the clinical test is the likelihood of a patient harboring high-grade PCa upon biopsy.

\section{Analytical specificity}

The specificity of the mRNA-based assays was determined both theoretically and analytically, by assessing the crossreactivity. The theoretical specificity was investigated using the NCBI's Basic Local Alignment Search Tool (BLAST) (version 2.3.0) tool. Sequences of primers, probes and complete PCR amplicons of the targets $D L X 1$, HOXC6 and KLK3 were checked for homology to pseudogenes, non-target related sequences or sequences of other organisms present in the GenBank ${ }^{\circ}$ database. The individual sequences of DLX1, HOXC6 and KLK3 and the amplicon sequences were tested using search sets "Human 
genomic + transcript" and "Others", including bacteria, viruses and other organisms, using programs MEGABLAST and BLASTN. Human genomic sequences and other organism sequences were evaluated looking for potential binding from the target sequences of the primers and probes using the Primer-BLAST program.

Cross-reactivity was tested at least quadruplicate using genomic DNA of eight bacterial and one yeast species known to be commonly involved in urinary tract infections. Bacteriuria as result of acute cystitis or acute pyelonephritis is defined as the presence of $110^{3}$ or more colony forming units (CFU) per $\mathrm{mL}$ of urine. Based on the yield of the bacterial cultures, $110^{5}$ copies DNA/PCR were included for Staphylococcus saprophyticus, Enterococcus faecium and Escherichia coli, while for Klebsiella pneumoniae subspecies pneumonia, Pseudomonas aeruginosa, Proteus mirabilis and Enterobacter aerogenes $1 \cdot 10^{7}$ copies DNA/PCR were used. Staphylococcus aureus and the yeast strain Candida albicans were tested at both $1110^{5}$ copies DNA/PCR and $1110^{7}$ copies DNA/PCR. In addition, RNase-treated human genomic DNA was evaluated at $20 \mathrm{ng} / \mathrm{PCR}$.

\section{Analytical sensitivity}

For each of the targets DLX1, HOXC6 and KLK3 serial dilution series of IVT RNAs were made in carrier RNA diluent (Labo Biomedical Products B.V., Rijswijk, the Netherlands) (12 $\mathrm{ng} / \mu \mathrm{l})$, resulting in specimens with IVT RNA concentrations of 640,320, 160, 80, 40, 20 and 10 copies per RT-PCR reaction. To assess the limit of detection (LOD) and the lower limit of quantification (LLOQ) of the DLX1, HOXC6 and KLK3 assays, 12 replicates of each IVT dilution were tested in 4 individual RT-PCR experiments. The LOD was determined as the lowest IVT concentration that could be detected in $\geq 95 \%$ of the samples. The LLOQ was determined as the lowest IVT concentration that could be measured with acceptable accuracy, determined as a standard deviation (SD) $<0.5$ Cp.

\section{Linearity}

To determine linearity, specimens were used with $D L X 1$, HOXC6 and KLK3 IVT RNA concentrations of $10^{7}, 10^{6}$, $10^{5}, 10^{4}, 10^{3}, 10^{2}$ copies per RT-PCR reaction. The lowest point was defined as $20 \%$ below LLOQ resulting in 16 copies/RT-PCR for DLX1 and KLK3 and 32 copies/ RT-PCR for HOXC6. For each target, six replicates of each concentration were tested in 5 individual RT-PCR experiments. Linearity was assessed using the polynomial method by fitting quadratic and cubic regression models and comparing whether these models were a better fit than linear regression [14]. The assays were considered linear if the cubic and quadratic variables for a $2^{\text {nd }}$ or $3^{\text {rd }}$ order polynomial were not statistically significant $(P>0.05)$ [15]. If either one of these higherorder terms failed to meet the linearity criterion, the deviation from linearity (DLi) was assessed using a weighted linear regression of the normalized $\mathrm{Cp}$ values versus the logarithm of the copy-numbers for each input concentration. If the absolute maximum DLi across concentrations was $\leq 0.5 \mathrm{Cp}$, the deviation was considered minimal and linearity was established for the assay.

\section{Precision}

Precision assessment included PCR instruments, interrun, operator and intra-run variation. The overall precision of the assays was determined for DLX1, HOXC6 and KLK3 using three dilutions of IVT RNA's with carrier RNA diluent (12ng/ $\mu \mathrm{l}$ ) (Labo Biomedical Products B.V., Rijswijk, the Netherlands). The lowest copy numbers were defined as $2 x(L L O Q+3 x \mathrm{SD})$, the other concentrations were based on $5 \mathrm{x}$ and 20x LLOQ. This resulted in specimen with $D L X 1$ concentrations of 400 , 100 and 76 copies per RT-qPCR, KLK3 concentrations of 400, 100 and 67 copies per RT-PCR and 800, 200 and 125 copies of HOXC6 per RT-PCR. Eight replicates of each IVT concentration were tested using three LightCycler 480 II instruments with a 384-well plate format (Roche Life Science, Indianapolis, IN, USA), by 3 operators on 3 consecutive days. Specific sub-analyses of this dataset allowed the analysis of the intra-run (repeatability), interrun precision (reproducibility), inter-instrument and interoperator precision, with the other factors being fixed.

Precision assessment of the combination of MagNA Pure 96 Instrument and LightCycler ${ }^{\circ} 480$ II instrument (Roche Life Science, Indianapolis, IN, USA) was done using urine samples of anonymous healthy males, stabilized in stabilization buffer as previously described. For this purpose, $2000 \mathrm{LNCaP}$ cells $/ \mathrm{mL}$ were added to the urine after which the samples were distributed into 4 aliquots of $1.1 \mathrm{~mL}$ and stored at $-80{ }^{\circ} \mathrm{C}$. RNA extraction and PCR amplification were performed in triplicate by 2 operators on 4 days.

All assays were considered precise if the $\mathrm{SD} \leq 0.5 \mathrm{Cp}$. The reproducibility and robustness of the assays were further assessed during an external precision experiment, in which a total of 99 post-DRE urine specimens were tested side-by-side study in two independent laboratories (Lab A and Lab B). Beforehand, it was defined that between laboratories the urine test outcome in terms of absolute difference in likelihood for high-grade PCa upon biopsy was not allowed to be more than $2 \%$.

\section{Interference testing}

To study the potential loss of assay sensitivity due to interfering endogenous substances that can occur in urine, e.g. uric acid, hemoglobin, white blood cells, red blood cells, albumin, bilirubin and human IgG, high 
concentrations of these potential interfering substances were added to urine (Table 2). For this purpose, mock urine samples were prepared using pooled healthy donor urine spiked with the biomarker-positive cell line LNCaP at two concentrations. In total, two urine samples containing 20,000 cells $/ \mathrm{mL}$ and two urine samples containing 10,000 cells $/ \mathrm{mL}$ were tested in duplicate.

Westgard et al. defined guidelines for the interpretation of a control result that is $1 \mathrm{SD}, 2 \mathrm{SD}$, or 3SD from the mean [16]. The average SD of 0.3 used in this analysis was obtained from the precision experiments done for the instrument combination. The Westgard rules were used for data analysis of the interference study with a difference in $\mathrm{Cp}$ value of less than $1 \mathrm{SD}$ scored 0 (within the 67\% confidence interval (CI)), less than $2 \mathrm{SD}$ scored 1 (within the 95\% CI), less than 3 SD (within the 99\% CI) scored 2 and differences larger than $3 \mathrm{SD}$ scored 3 . When using a specific candidate interfering substance, multiple scores of 2 and 3 were considered inhibition.

\section{Effect of the stabilization buffer on clinical performance} The performance of the stabilization buffer was validated through a direct comparison of the RNA value obtained from urine samples collected in the stabilization buffer from a cohort of 99 men scheduled for prostate biopsies and the RNA value obtained from samples in Progensa ${ }^{\circ}$ urine transport medium (Hologic Inc., San Diego, CA, USA). The latter data were obtained from two published, independent prospective multicenter studies, clinical study A ( $n=519$ samples) and B ( $n=386$ samples) [10].

\section{Stabilization buffer stability}

The stability of urine specimens in the stabilization buffer was evaluated by processing urine of five patients

Table 2 Test concentrations versus normal concentration of endogenous substances in urine

\begin{tabular}{|c|c|c|}
\hline Urine Constituents & Test concentration & $\begin{array}{l}\text { Normal concentration } \\
\text { in urine }\end{array}$ \\
\hline Hemoglobin & $2 \mathrm{mg} / \mathrm{mL}^{\mathrm{a}}$ & $<3 \mu \mathrm{mol} / \mathrm{L}$ \\
\hline Uric Acid & $0.235 \mathrm{mg} / \mathrm{mL}$ & $\begin{array}{l}0.034-0.070 \mathrm{mg} / \mathrm{mL} \\
\text { (male) }\end{array}$ \\
\hline Human lgG & $3 \mathrm{mg} / \mathrm{mL}$ & $<6 \mu \mathrm{g} / \mathrm{mL}$ \\
\hline Bilirubin & $0.12 \mathrm{mg} / \mathrm{mL}$ & $\begin{array}{l}\text { not present in the } \\
\text { urine of normal, } \\
\text { healthy individuals. }\end{array}$ \\
\hline Albumin & $\begin{array}{l}1 \mathrm{mg} / \mathrm{mL} \text { (Albuminuria } \\
\text { criteria) }\end{array}$ & $<30 \mathrm{mg} / 24 \mathrm{~h}$ \\
\hline Leukocytes & $4.5 \times 10^{4}$ cells $/ \mathrm{mL}$ & $\begin{array}{l}<10 \text { leukocytes/high } \\
\text { powered field }\end{array}$ \\
\hline $\begin{array}{l}\text { Red blood cells } \\
\text { (RBC) }\end{array}$ & $3.0 \times 10^{4}$ cells $/ \mathrm{mL}$ & $\begin{array}{l}<3 \mathrm{RBC} / \text { high powered } \\
\text { field }\end{array}$ \\
\hline
\end{tabular}

$R B C$ red blood cells

${ }^{\mathrm{a}}$ This test concentration is far higher than typically expected in clinical practice incubated at $4{ }^{\circ} \mathrm{C}$, room temperature and $30{ }^{\circ} \mathrm{C}$ up to 15 days after collection. From the start, all samples were split into two aliquots and analyzed in duplicate after which RNA extraction, RT-PCR and RNA value calculation were performed.

The influence of transit time on RNA value was determined using the 99 samples processed in the stabilization buffer. The number of samples received on Day 1 (day of collection) and Days 2, 3, 4-6 were 56, 16, 17 and 10 respectively. Furthermore, the effect of decline in RNA value due to RNA degradation on the likelihood score for highgrade PCa was studied. The 56 samples received at the day of collection (Day 1) were used for this analysis as these samples were considered to be least affected by RNA degradation. Additionally, the RNA stability was tested after 1-year storage at $-80{ }^{\circ} \mathrm{C}$ using the 99 samples processed in the stabilization buffer.

\section{Statistical analysis}

Statistical analyses were performed with SPSS v.20.0 (IBM Corp., Armonk, NY, USA) and R v.3.2.1 (R Foundation for Statistical Computing, Vienna, Austria). Because calculated RNA values are continuously increasing with patient risk, the performance was assessed and evaluated as area under the curve (AUC) of the receiver operating characteristic (ROC). The 95\% confidence intervals and comparisons of AUCs were determined using DeLong's method as implemented in the $\mathrm{R}$ package pROC [17]. The non-parametric Wilcoxon signed-rank test was used to compare the results obtained for 99 urine samples tested in Lab A and Lab B. Analysis of Variance (ANOVA) and the Kruskal-Wallis test were used to test for differences between the medians of RNA values over transit days at room temperature. For each comparison between two groups, if $P<0.05$, the conclusion is that they differ significantly from each other.

\section{Results}

\section{Analytical specificity}

BLAST searches revealed that the target sequences of $D L X 1, H O X C 6$ and KLK3 showed no homology to the DNA of bacteria, viruses, other organisms or other human DNA or RNA sequences. Cross-reactivity experiments were conducted to determine the practical specificity, using human genomic DNA and genomic bacterial DNA as template for the mRNA-based assays. No cross-reactivity was observed for human genomic DNA and most bacterial strains and yeast strain as obtained Cps were below the LOD. Some genomic bacterial DNA's resulted in a very low signal for KLK3 only, with the highest result for Enterobacter aerogenes (max. 113 copies). However, the amplification curves of this bacterial strain had an aberrant pattern and analysis of the PCR products using a TapeStation instrument (Agilent 
Technologies Inc., Santa Clara, CA, USA) confirmed that the signal obtained was non-specific (data not shown). Even if the signal cannot be separated from a true positive one, it would account for only $<1 \%$ of the reference gene $K L K 3$ and the impact on test results can be considered negligible.

\section{Analytical sensitivity}

The analytical performance of the mRNA-based assays was assessed and the established limits of detection and quantification are depicted in Table 3. The LLOQ for $K L K 3$ and $D L X 1$ was determined at 20 copies/RT-PCR and the LLOQ for HOXC6 was determined at 40 copies/ RT-PCR. However, repeat experiments to verify this difference of LLOQ between HOXC6 versus KLK3 and $D L X 1$, showed that an LLOQ of 20 copies/RT-PCR could also be established for HOXC6. All assays had LODs ranging from $\mathrm{Cp}$ 35.97-38.26, corresponding to 10 copies of target RNA. The LLOQs ranged from Cp $35.20-36.01$, indicating that all assays can be accurately quantified from 20 copies of target RNA upward. For all RT-PCR assays the ULOQ was $10^{7}$ copies/RT-PCR, as determined in the linearity experiment, establishing a working range of 10 to $10^{7}$ copies for all genes.

\section{Linear range}

Both the DLX1 and HOXC6 mRNA-based assays were linear over the entire range of dilutions as established by the non-significant coefficients of the cubic and quadratic variables $(P>0.05)$ in the second and third order polynomial regression analyses (Table 4 ). The $\mathrm{Cp}$ values ranged from 16.74 to 36.49 (corresponding with $10^{7}-16$ copies/RT-PCR) for $D L X 1$, and from 16.40 to 36.30 (corresponding with $10^{7}-32$ copies/RT-PCR) for HOXC6.

Since the cubic and quadratic variables were statistically significant for $K L K 3 \quad(P<0.001)$, this assay was not considered linear when all seven concentrations were taken into account (Table 4). If only five dilutions with $\geq 1000$ copies $K L K 3 /$ RT-PCR were included, the assay was linear. To assure sufficient input material for the clinical assay, a minimum of 10,000 copies of KLK3 need to be present. Hence, the assay is linear in the range of its clinical performance.

The deviation from linearity (DLi) was assessed for the KLK3 mRNA-based assay. When all seven concentrations were taken into account, the DLi was within the preset limits and hence, the $K L K 3$ assay was considered sufficiently linear, even when fewer than 1000 copies were present (Fig. 1).

In summary, all mRNA-based assays can accurately quantitate their RNA target sequence over the entire evaluated range of concentrations, encompassing those that can occur in whole urine specimens.

\section{Precision}

Precision of the mRNA-based assays was evaluated under various conditions covering inter-run, PCR instrument and operator variation. The average $\mathrm{Cp}$ and SD $\mathrm{Cp}$ of the results are shown in Table 5. The overall precision was determined by testing the reproducibility of a sample tested at three concentrations in 8 replicates between three operators, three instruments and 3 days resulting in $\mathrm{SD}$ of the Cp ranging from 0.1 to 0.3 .

Intra-run precision was based on 8 data points per concentration, generated by one operator on the same day and on the same machine resulting in SD of the $\mathrm{Cp}$ ranging from 0.1 to 0.5 . Inter-run precision was based on 24 data points per concentration, generated by one operator on three different days, with the SD of the $\mathrm{Cp}$ ranging from 0.1 to 0.4 . Inter-instrument precision was based on the 24 data points per concentration, generated on three different PCR instruments by one operator on the same day, resulting in $\mathrm{SD}$ of the $\mathrm{Cp}$ ranging from 0.1 to 0.4. Finally, inter-operator precision was based on 24 data points per concentration, generated by three different operators on the same day and on the same PCR instrument, resulting in $\mathrm{SD}$ of the Cp ranging from 0.1 to 0.3.

The precision of MagnaPure and LightCycler ${ }^{\circ} 480$ II Instruments combination was based on 33 data points generated by two operators on four different days using aliquots of urine mixed with $2000 \mathrm{LNCaP}$ cells $/ \mathrm{mL}$. An average Cp of 22.7 with an SD of $0.3 \mathrm{Cp}$ was obtained for $K L K 3$, a Cp of 28.2 with an SD of 0.3Cp for HOXC6 and a Cp of 31.9 with an SD of 0.3Cp for DLX1. All precision measurements demonstrated a robust analytical performance, with overall precision $\mathrm{SD}$ of maximum $0.3 \mathrm{Cp}$ being within the acceptance criteria of $\leq 0.5$.

\section{Interference testing}

The most common urinary substances were evaluated for potential interference with the actual assay outcome, i.e. hemoglobin, uric acid, bilirubin, albumin, white blood

Table 3 The analytical measurement range of the assays for DLX1, HOXC6 and KLK3

\begin{tabular}{llll}
\hline Target & LOD in copies/RT-PCR (Avg Cp) & LLOQ in copies/RT-PCR (Avg Cp) & ULOQ in copies/RT-PCR (Avg Cp) \\
\hline DLX1 & $10(37.24)$ & $20(36.00)$ & $10^{7}(16.74)$ \\
HOXC6 & $10(38.26)$ & $20(36.01)$ & $10^{7}(16.40)$ \\
KLK3 & $10(35.97)$ & $20(35.20)$ & $10^{7}(16.74)$ \\
\hline
\end{tabular}

Avg average, Cp crossing point, DLX1 Distal-less Homeobox 1, HOXC6 Homeobox C6, KLK3 Kallikrein-3, LOD Limit of Detection, LLOQ Lower Limit of Quantification, $R T-P C R$ reverse-transcriptase polymerase chain reaction, ULOQ Upper Limit of Quantification 
Table $4 P$-values associated with the polynomial regression coefficients for the dilution series

\begin{tabular}{llllll}
\hline Regression Type & Coefficient & DLX1 & HOXC6 & KLK3 (7 concentrations) & KLK3 (5 concentrations) \\
\hline $2^{\text {nd }}$ order & Quadratic P-value & 0.7 & 0.7 & $<0.001$ & 0.7 \\
$3^{\text {rd }}$ order & Quadratic P-value & 0.3 & 0.9 & $<0.001$ & 0.8 \\
& Cubic P-value & 0.3 & 0.9 & $<0.001$ & 0.8 \\
\hline
\end{tabular}

DLX1 Distal-less Homeobox 1, HOXC6 Homeobox C6, KLK3 Kallikrein-3

cells, red blood cells and monoclonal immunoglobulins such as human IgG and the Westgard scores are shown in Table 6.

Most constituents were shown to be no inhibitors of the assays. However, in half of the samples studied, hemoglobin resulted in Westgard scores of 2 for DLX1 and HOXC6. While there is typically no free hemoglobin present in urine, it appears in urine of patients with hemoglobinuria due to e.g. acute glomerulonephritis, kidney cancer, malaria or pyelonephritis, giving urine a purple color. Red blood cells will be absent in urine of patients with hemoglobinuria, in contrast to hematuria, as a result of urinary tract infections or bladder cancer. Although intact red blood cells do not impact the test results, free hemoglobin due to damaged red blood cells could act as an inhibitor.

\section{Clinical performance bridging study}

The clinical performance of the mRNA-based assays was validated through an external precision experiment, performed in an independent laboratory. To this purpose, RNA was extracted from 99 whole urine samples collected post-DRE. Sample validity was based on KLK3 in the sample, which can be considered as a measure for the presence of cellular or exosome content originating from the prostate, and the LOD of the DLX1 and HOXC6 mRNA assays. The decision tree to determine sample validity is shown in Fig. 2.

The number of valid samples was 99 resulting in an informative rate of $100 \%$. The inter-laboratory variation was determined for each mRNA-based assay. Per laboratory, the $\mathrm{Cp}$ value of the sample was subtracted from the $\mathrm{Cp}$ value obtained for the calibrator of each target. This was only done for $C p$ values $\leq L L O Q$, since these can be accurately quantified. For each sample the difference between the Cp values obtained at Lab A and Lab B was calculated per target (Fig. 3).

Non-inferiority testing showed that the absolute difference between the $\mathrm{Cp}$ values was not significantly higher than the $0.5 \mathrm{Cp}$ defined in the precision experiments as the maximum variation of the mRNA-based assays $(P=0.8$ for $D L X 1, P=0.1$ for HOXC6 and $P=1.0$ for $K L K 3$ ), showing robust performance of the test between laboratories.

The diagnostic performance of the mRNA-based assays for DLX1, HOXC6 and KLK3 was determined using the RNA value obtained for these 99 samples analyzed independently at Lab A and Lab B. The ROC was used to visualize the diagnostic efficacy of the RNA value for

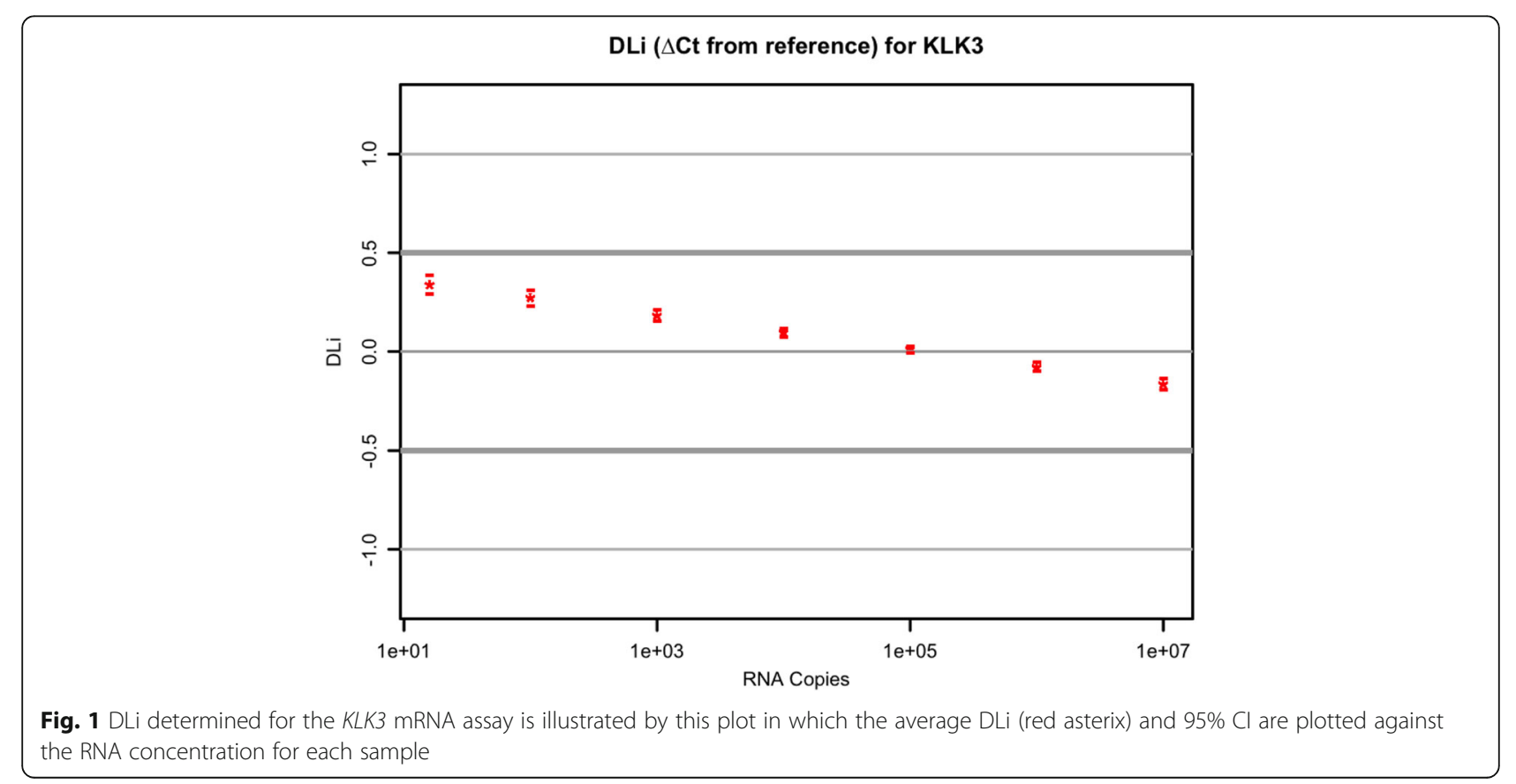


Table 5 Precision of $D L X 1, H O X C 6$, and KLK3 mRNA-based assays

\begin{tabular}{|c|c|c|c|c|c|c|c|c|c|c|}
\hline & \multirow[b]{2}{*}{ Copies/RT-PCR } & \multicolumn{3}{|l|}{ DLX1 } & \multicolumn{3}{|c|}{ HOXC6 } & \multicolumn{3}{|l|}{ KLK3 } \\
\hline & & 400 & 100 & 76 & 800 & 200 & 125 & 400 & 100 & 67 \\
\hline \multirow[t]{2}{*}{ Overall precision } & Avg Cp & 31.7 & 33.8 & 34.3 & 31.2 & 33.4 & 34.1 & 31.3 & 33.3 & 33.8 \\
\hline & SD Cp & 0.2 & 0.2 & 0.3 & 0.1 & 0.2 & 0.3 & 0.1 & 0.2 & 0.3 \\
\hline \multirow[t]{2}{*}{ Intra-run precision (repeatability) } & Avg Cp & 31.6 & 33.8 & 34.2 & 31.1 & 33.4 & 34.1 & 31.3 & 33.2 & 33.4 \\
\hline & SD Cp & 0.1 & 0.3 & 0.3 & 0.1 & 0.2 & 0.2 & 0.1 & 0.3 & 0.5 \\
\hline \multirow[t]{2}{*}{ Inter-run precision (reproducibility) } & Avg Cp & 31.8 & 33.8 & 34.4 & 31.2 & 33.4 & 34.1 & 31.4 & 33.4 & 33.9 \\
\hline & SD Cp & 0.1 & 0.3 & 0.4 & 0.1 & 0.2 & 0.2 & 0.2 & 0.2 & 0.4 \\
\hline \multirow[t]{2}{*}{ Inter-instrument precision } & Avg Cp & 31.7 & 33.9 & 34.3 & 31.2 & 33.4 & 34.2 & 31.4 & 33.4 & 33.9 \\
\hline & SD Cp & 0.1 & 0.2 & 0.4 & 0.1 & 0.3 & 0.2 & 0.1 & 0.2 & 0.3 \\
\hline \multirow[t]{2}{*}{ Inter-operator precision } & Avg $C p$ & 31.7 & 33.8 & 34.5 & 31.1 & 33.4 & 33.9 & 31.4 & 33.4 & 33.9 \\
\hline & SD Cp & 0.1 & 0.2 & 0.3 & 0.3 & 0.2 & 0.2 & 0.1 & 0.2 & 0.3 \\
\hline
\end{tabular}

Avg average, $C p$ crossing point, DLX1 Distal-less Homeobox 1, HOXC6 Homeobox C6, KLK3 Kallikrein-3, SD standard deviation

the detection of high-grade PCa. The AUC of the ROC obtained at Lab A (AUC $=0.71$ (95\% CI:0.60-0.81)) was not statistically significant different $(P=0.2)$ from the AUC obtained at Lab B (AUC $=0.73$ (95\% CI:0.62-0.83)).

To further validate the robustness of the assays between both laboratories, the outcome of the previously published logistic regression prediction model comprising the RNA values and traditional clinical risk factors i.e. PSA, prostate volume, DRE and family history, was used as validation [10]. A strong correlation coefficient $(r=0.997)$ was obtained between both laboratories as shown in Fig. 4, and this correlation was shown to be significant $\left(P=2.2 \cdot 10^{-16}\right)$. The clinical performance of the likelihood scores for the detection of high-grade $\mathrm{PCa}$ at Lab A (AUC $=0.92$ (95\% CI:0.84-0.99)) was not statistically significant different $(P=0.3)$ from the clinical performance at Lab B (AUC $=0.92$ (95\% CI:0.85-0.99). These results are in line with the clinical performance (AUC $=0.90$ (95\% CI:0.85-0.95)) obtained in the validation study recently described by Van Neste et.al. [10].

The absolute difference between the results in terms of likelihood for high-grade PCa upon biopsy was shown not to be significantly higher than $2 \%(P=1.0)$, showing robust performance of the test between laboratories.

\section{Clinical performance stabilization buffer}

To avoid RNA degradation, the samples should be stabilized immediately after urine collection and for this purpose

Table 6 Westgard scores for interference testing

\begin{tabular}{|c|c|c|c|c|c|c|c|}
\hline \multirow[b]{3}{*}{ Urine } & \multirow[b]{3}{*}{ Constituent } & \multicolumn{6}{|c|}{ Westgard Score } \\
\hline & & \multicolumn{3}{|c|}{ Sample 1} & \multicolumn{3}{|c|}{ Sample 2} \\
\hline & & $D L X 1$ & HOXC6 & $K L K 3$ & $D L X 1$ & HOXC6 & KLK3 \\
\hline \multirow[t]{7}{*}{ spiked with 20.000 LNCap cells/mL } & Uric Acid & 0 & 0 & 0 & 0 & 0 & 0 \\
\hline & Bilirubin & 0 & 0 & 0 & 0 & 0 & 0 \\
\hline & Albumin & 0 & 0 & 0 & 0 & 0 & 0 \\
\hline & Hemoglobin & 0 & 0 & 0 & 2 & 1 & 1 \\
\hline & Human IgG & 0 & 0 & 0 & 0 & 0 & 0 \\
\hline & Red blood cells (RBC) & 0 & 0 & 0 & 0 & 0 & 0 \\
\hline & Leukocytes & 0 & 0 & 0 & 0 & 0 & 0 \\
\hline \multirow[t]{7}{*}{ spiked with 10.000 LNCap cells/mL } & Uric Acid & 0 & 0 & 0 & 0 & 0 & 0 \\
\hline & Bilirubin & 0 & 0 & 0 & 0 & 0 & 0 \\
\hline & Albumin & 0 & 0 & 0 & 0 & 0 & 0 \\
\hline & Hemoglobin & 0 & 0 & 0 & 2 & 2 & 1 \\
\hline & Human lgG & 0 & 1 & 1 & 1 & 0 & 1 \\
\hline & Red blood cells (RBC) & 0 & 0 & 0 & 0 & 0 & 0 \\
\hline & Leukocytes & 0 & 0 & 0 & 0 & 0 & 0 \\
\hline
\end{tabular}




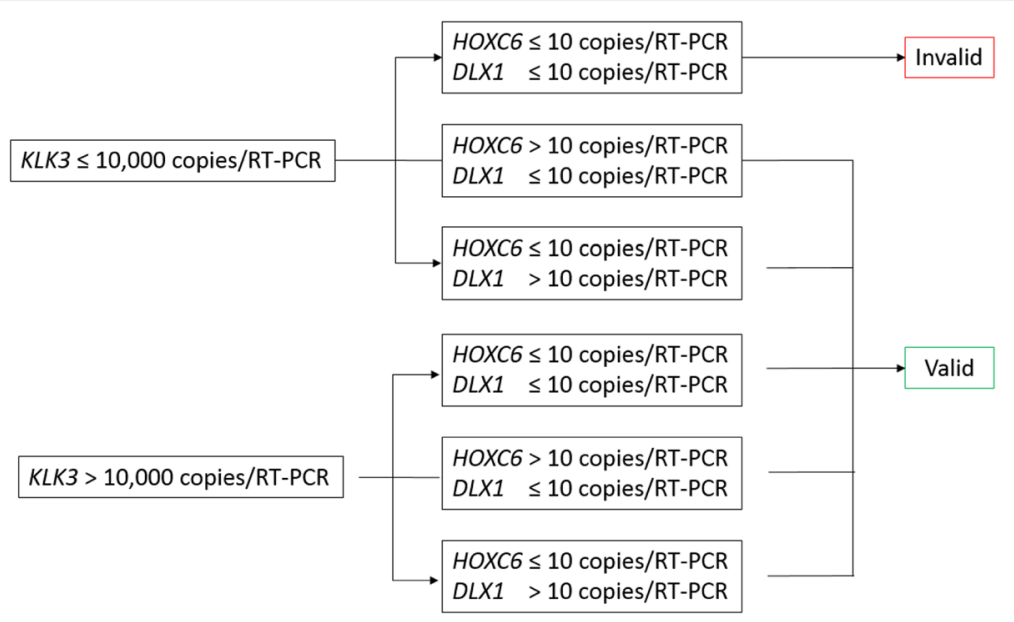

Fig. 2 Decision tree to determine sample validity

a stabilization buffer was developed. Assay performance for the prediction of high-grade PCa upon biopsy using samples collected in this buffer (Clinical trial C: AUC 0.71 (95\%CI: $0.60-0.81$ ) was comparable to the performance using samples collected in Progensa ${ }^{\circ}$ urine transport medium (Clinical Trial A: AUC 0.76 (95\%: 0.71-0.81) and Clinical trial B: AUC 0.73 (95\%: 0.67-0.78)), as indicated by the non-significant differences in AUC's (Trial A versus C; $P=0.4$, Trial B versus $C ; P=0.8$ ).

\section{Stabilization buffer and assay robustness}

To determine the maximum allowable transport conditions for sample transfer from clinic to the laboratory, the

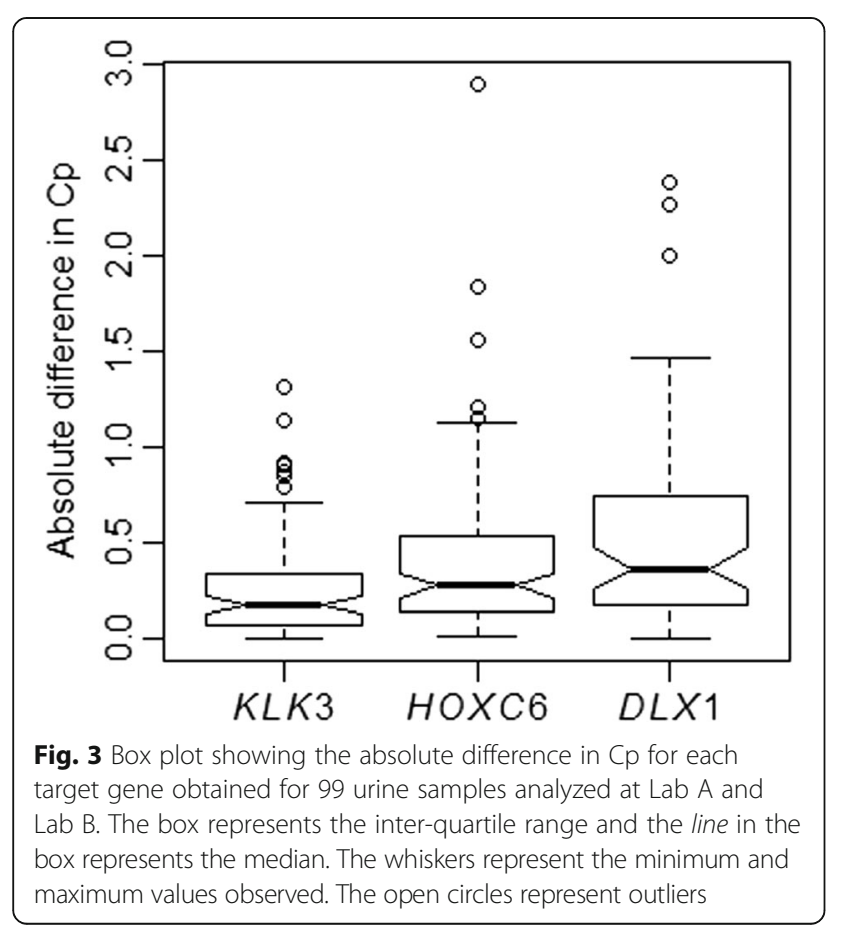

robustness of the RNA value was evaluated over a period of 2 weeks at $4{ }^{\circ} \mathrm{C}$, room temperature and $30{ }^{\circ} \mathrm{C}$. The RNA values were calculated relative to the average RNA value obtained on Day 1 (Fig. 5). Until Day 5 from collection, there was no significant signal degradation for samples stored at room temperature and $4{ }^{\circ} \mathrm{C}(P=0.5$ for storage duration and $P=0.6$ for storage temperature).

Post-DRE collected whole urine in stabilization buffer resulted in max. $15 \%$ decrease of RNA value up to 4 days after collection at either $4^{\circ} \mathrm{C}$ or room temperature. At $30{ }^{\circ} \mathrm{C}$, the $15 \%$ decrease in RNA value was already obtained on Day 2 from collection. Temperature had a significant

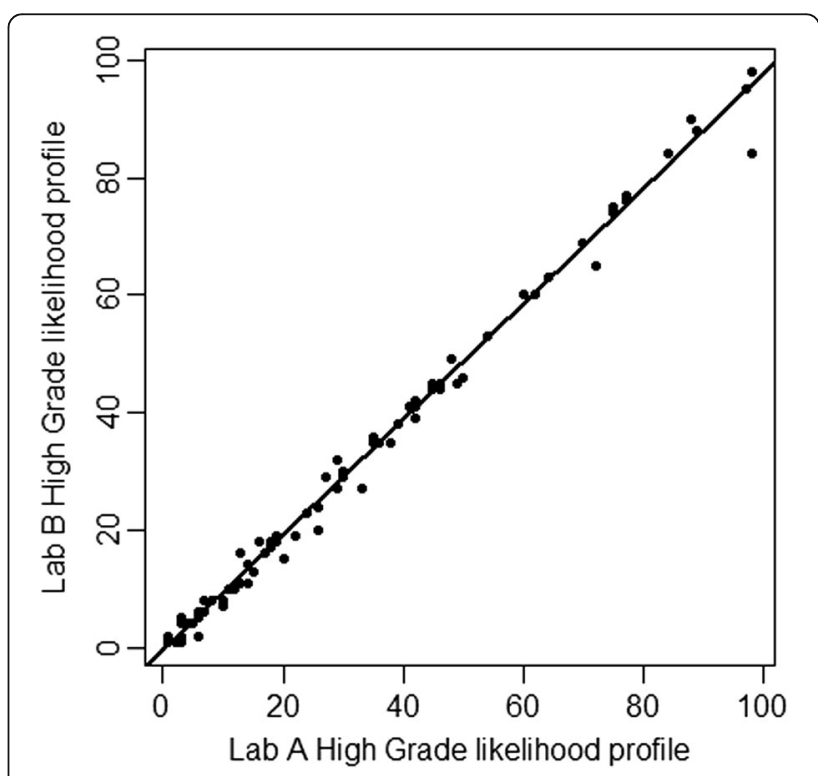

Fig. 4 Scatter plot showing the correlation between the likelihood profiles for high-grade PCa upon biopsy obtained for 99 urine samples analyzed at Lab. A and Lab. B with $y=0.982, r=0.997$ and $P<0.001$ 


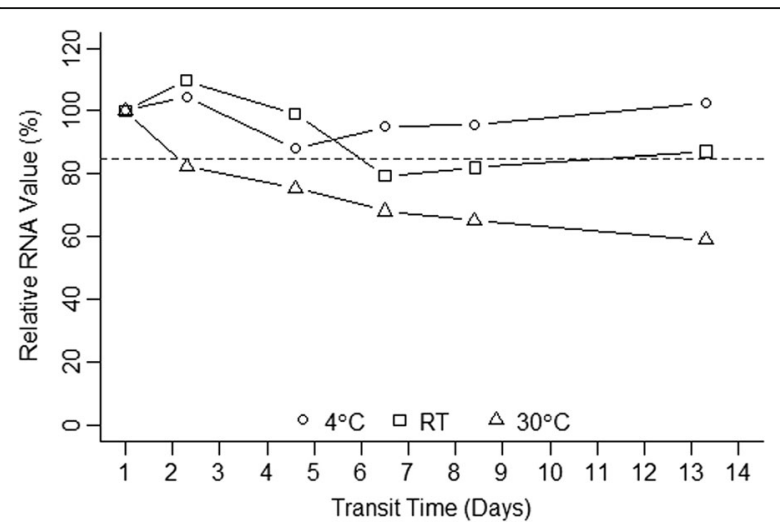

Fig. 5 Influence of temperature and time on RNA value using samples collected in stabilization buffer. The influence of RNA degradation on RNA level should not be greater than $15 \%$ as indicated by dotted line at $85 \%$

effect on the RNA value for longer storage periods $(P<0.05)$. However, up to Day 4 from collection, neither temperature $(P=0.1)$ nor storage duration $(P=0.3)$ had a significant effect on the RNA value.

The influence of transit time on RNA value was also determined using the 99 evaluable urine samples in stabilization buffer (Fig. 6) and was shown to be minimal as no significant differences $(P=0.9)$ were observed between the medians of RNA values obtained for Day 1 (56 samples) through Day 4 (10 samples).

The influence of RNA degradation on the likelihood for high-grade PCa was determined. Using the 56 samples collected on Day 1, the influence of $15 \%$ RNA value decrease on the likelihood for high-grade PCa was calculated and resulted in $\leq 2 \%$ change in likelihood score for high-grade $\mathrm{PCa}$.

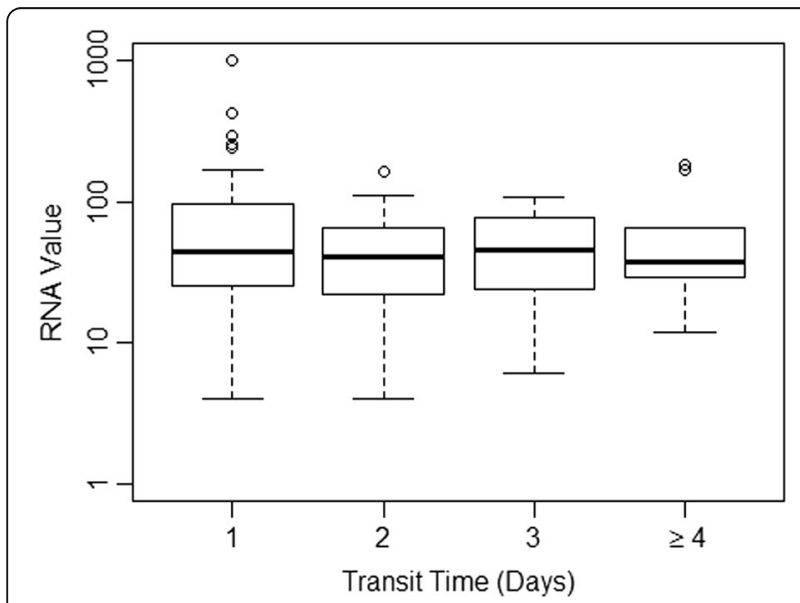

Fig. 6 Boxplot summarizing the influence of time on RNA value using samples collected in stabilization buffer. The box represents the inter-quartile range and the line in the box represents the median. The whiskers represent the minimum and maximum values observed
Finally, the long-term stability was tested before and after a storage period of 1 year at $-80{ }^{\circ} \mathrm{C}$. The clinical performance using RNA values obtained immediately after urine collection (AUC 0.72(95\% CI: 0.61-0.83)) and after 1-year storage (AUC 0.71 (95\% CI: 0.60-0.81)) was not affected, as illustrated by the non-significant difference between the AUC's $(P=0.5)$.

\section{Discussion}

The major challenge in PCa detection and management is to differentiate aggressive from non-aggressive cancer at an early stage of this disease. Currently, PCa diagnosis is driven by PSA, fostering overdiagnosis and overtreatment of indolent tumors. A test that would better separate men with high-grade $\mathrm{PCa}$ from those with indolent or no disease is urgently needed. Previously, it was shown that high-grade $\mathrm{PCa}$ could be detected using a urinary biomarker-based risk score [10]. This test, based on two mRNA assays for the DLX1 and HOXC6 biomarkers, was developed on the LightCycler 480 II platform for the specific detection of high-grade $\mathrm{PCa}$ in urinary samples. $K L K 3$ transcripts, considered as a measure for the presence of content from prostatic origin, i.e. cells or exosomes, was used as reference marker to normalize the DLX1 and HOXC6 signals. In men considered for biopsy, e.g. due to elevated PSA, the RNA value combined with other clinical risk factors led to an improved patient risk stratification for high-grade PCa [10].

Although clinical validity is of obvious importance, analytical validity and ease of use are essential for the successful implementation of novel diagnostic tests into clinical practice. In this report, we have described the stability testing of mRNA using a stabilization buffer and a simplified urine sample collection method, and demonstrated the robust analytical performance of the quantitative mRNA assays.

An important aspect of this study is the comparison of the clinical performance characteristics of the mRNAbased assays between two independent laboratories using whole urine collected post-DRE from a cohort of 99 men considered for prostate biopsy. For each target it was shown that the absolute difference in Cp per sample was not significantly higher than the maximum variation obtained in the precision studies for the assays $(0.5 \mathrm{Cp})$. This is an important aspect assuring assay robustness, as it implicates that the variation introduced by different MagNa Pure 96 instruments and different laboratories does not significantly affect the overall precision or influence the patient's result. Whole urine collected postDRE from a cohort of 99 men considered for prostate biopsy showed no statistical difference in clinical performance between two laboratories. Furthermore, an absolute difference between the results in terms of likelihood for high-grade PCa upon biopsy was shown to 
be significantly lower than $2 \%$, demonstrating the robust performance of both the standardized automated RNA extraction and RT-PCR assays.

Overall, the mRNA-based assays for DLX1, HOXC6 and $K L K 3$ exhibited a precise and robust behavior, with the natural variation or experimental noise being the main factor influencing the outcome. The variation introduced by the operator, different runs, PCR instrument or even the combination of automated RNA extraction and PCR platform (96 well versus 384 well format), RNA extraction instrument and different laboratory settings was limited and considered well within the allowable limits. The assay results were accurate and reproducible for the quantification of expression levels of the three targets over a wide range of concentrations, covering the typical content of a post-DRE whole urine sample. This illustrates that the mRNA-based assays are suitable for clinical testing, not only in routine or reference laboratories, but also in general molecular pathology laboratories.

High quality urine samples are key to minimize potential bias in results. Therefore, no more than $30 \mathrm{ml}$ first catch urine should be collected after a six-stroke DRE in order to prevent false negative results due to a dilution effect. To avoid RNA degradation, a stabilization buffer was developed allowing temporary preservation of the mRNA in whole urine and resulting in a high rate of evaluable samples under routine collection conditions. Easy sample shipment from clinics to laboratories is feasible as the RNA of urine samples in this stabilization buffer is stable at room temperature up to 5 days from collection, assuring standardized, reproducible and reliable results. Using this buffer, the clinical performance was comparable to the test performance shown in two independent clinical studies using Progensa ${ }^{\circ}$ urine transport medium. Furthermore, it was demonstrated that RNA in whole urine collected in this stabilization buffer was stable for 1 year at $-80{ }^{\circ} \mathrm{C}$, as the same clinical performance in terms of AUC was obtained.

The influence of inhibitory substances that may occur in urine on the assays was also evaluated. Although intact red blood cells in urine were not inhibitory, free hemoglobin was indicated as a possible inhibitor of the mRNA-based assays for HOXC6 and DLX1. Hemoglobin is known to potentially act as an inhibitor to PCR reactions [18]. The inhibition of hemoglobin on these assays was surprising, since the manufacturer of the PCR master mix specially recommended it for use with whole blood samples. However, the results should be interpreted with caution as the evaluated hemoglobin concentration hemoglobin $(2 \mathrm{mg} / \mathrm{ml})$ is the equivalent of $\sim 69,000,000$ lysed red blood cells containing 29 pg hemoglobin per cell [19]. This high concentration even caused clotting of the compound in urine sample 1 (Table 6) showing no inhibition. Such high concentrations of hemoglobin are only representative for patients with gross hematuria and patients with hemoglobinuria presenting with purple urine as result of the presence of free hemoglobin. Gross hematuria only occurs in 4 per 1000 urological patients, and has not been observed in over 1000 urine samples that were tested in clinical validation studies [10, 20]. In case of gross hematuria, intact red blood cells will contain most of the hemoglobin, hence free hemoglobin is not likely to pose a problem. Testing inhibition in urine samples of patients instead of inhibitory compounds, will yield a more accurate reflection of diagnostic test performance.

The automated RNA extraction and PCR platform used in this validation study contribute to the robust performance of the mRNA assays. Easy-to-use and efficient handling is provided by both platforms allowing standardized extraction and analysis of large numbers of samples. Since platform or reagent change might affect the clinical performance of the assays, it is recommended to routinely monitor the analytical characteristics using appropriate quality controls for each batch of samples analyzed, guaranteeing reliable results for the likelihood of high-grade PCa.

\section{Conclusion}

The analytical validation of the mRNA-based assays for $D L X 1, H O X C 6$ and $K L K 3$ indicate a precise and robust performance. A standardized whole urine sample collection procedure, a widely used automated RNA extraction and PCR platform contribute to the overall good performance of the assays. Results of this study illustrate the robustness of the assays, enabling testing in routine laboratories but also in general molecular pathology laboratories that have both platforms available. Independent clinical studies have shown that the RNA value can complement and improve upon the existing pre-biopsy risk assessment tools, such as PSA, to predict the likelihood of finding high-grade PCa upon biopsy. The combination of these mRNA-based assays with simple specimen processing will provide physicians with test results from a robust and reliable mRNA-based urine test.

\section{Abbreviations \\ AUC: Area under curve; CLIA: Clinical Laboratory Improvement Amendments; Cp: Crossing point; DLi: Deviation from linearity; DLX1: Distal-less Homeobox 1; DRE: Digital rectal examination; GS: Gleason score; HOXC6: Homeobox C6; IVT: In vitro transcribed RNA; KLK3: Kallikrein 3; NAI-: Nucleic acid isolation control; NTC: No-template control; PCa: Prostate cancer; PSA: Prostate-specific antigen; RT-PCR: Reverse transcriptase-polymerase chain reaction; $\triangle \triangle \mathrm{Cp}$ : Delta delta $\mathrm{Cp}$ method}

\section{Acknowledgements}

The authors would like to thank Shannon Dempsey, Gabriel Alvarado, Jane Pham and Dagoberto Gomez of MDxHealth Inc. for their contribution to the practical work and data analysis and Dr Jack Groskopf of MDxHealth Inc. for critically reading the manuscript, the urologists of the participating clinics and all patients that gave their consent. 
Funding

None.

\section{Availability of data and materials}

The datasets used and/or analyzed during the current study are available from the corresponding author on request.

\section{Authors' contributions}

$\mathrm{DH}$ participated in research design, participated in data analysis and writing of the paper. HdJ participated in research design, performance of the research and participated in data analysis. SJ participated in research design, performance of the research and participated in data analysis. MC participated in research design, performance of the research and participated in data analysis. MK participated in research design, performance of the research and participated in data analysis. WVC participated in research design and data analysis and was supervisor of the study. LVN participated in data analysis and writing of the paper. JS participated in research design, writing of the paper and was supervisor of the study. All authors read and approved the final manuscript.

\section{Competing interest}

The authors (DH,HdJ,SJ,MC,MK) declare that they are employees of MDxHealth. WVC, LVN, JS are consultants for MDxHealth.

\section{Consent for publication}

Not applicable.

\section{Ethics approval and consent to participate}

All participants signed a consent in accordance with the Declaration of Helsinki -based form that was approved by the institutional review board of the participating clinics (Radboud University Medical Center Nijmegen, CWZ Hospital Druten, St. Elisabeth Hospital Tilburg, Scheper Hospital Emmen, ZGT Hospital Hengelo, Slingeland Hospital Doetinchem).

\section{Publisher's Note}

Springer Nature remains neutral with regard to jurisdictional claims in published maps and institutional affiliations.

\section{Author details}

${ }^{1}$ MDxHealth B.V., Nijmegen, The Netherlands. ${ }^{2}$ MDxHealth Inc., Irvine, CA, USA. ${ }^{3}$ Department of Mathematical Modelling, Statistics and Bioinformatics, Ghent University, Ghent, Belgium. ${ }^{4}$ Department of Pathology, Maastricht University Medical Center, Maastricht, The Netherlands. ${ }^{5}$ Department of Urology, Radboud University Nijmegen Medical Centre, Nijmegen, The Netherlands.

Received: 20 April 2017 Accepted: 2 May 2017

Published online: 11 May 2017

\section{References}

1. Ferlay J, Steliarova-Foucher E, Lortet-Tieulent J, Rosso S, Coebergh JWW, Comber H, Forman D, Bray F. Cancer incidence and mortality patterns in Europe: estimates for 40 countries in 2012. Eur J Cancer. 2013;49:1374-403.

2. Siegel RL, Miller KD, Jemal A. Cancer statistics, 2016. CA Cancer J Clin. 2016; 66:7-30.

3. Torre LA, Bray F, Siegel RL, Ferlay J, Lortet-Tieulent J, Jemal A. Global cancer statistics, 2012. CA Cancer J Clin. 2015;65:87-108.

4. Yatani R, Chigusa I, Akazaki K, Stemmermann GN, Welsh RA, Correa P. Geographic pathology of latent prostatic carcinoma. Int J Cancer. 1982;29: 611-6.

5. Postma R, Schröder FH, van Leenders GJLH, Hoedemaeker RF, Vis AN, Roobol MJ, van der Kwast TH. Cancer detection and cancer characteristics in the European Randomized Study of Screening for Prostate Cancer (ERSPC)-Section Rotterdam. A comparison of two rounds of screening. Eur Urol. 2007;52:89-97.

6. Leyten GHJM, Hessels D, Smit FP, Jannink SA, de Jong H, Melchers WJG, Cornel EB, de Reijke TM, Vergunst H, Kil P, Knipscheer BC, de Kaa CAH-V, Mulders PFA, van Oort IM, Schalken JA. Identification of a Candidate Gene Panel for the Early Diagnosis of Prostate Cancer. Clin Cancer Res. 2015;21: 3061-70.

7. Altintas DM, Allioli N, Decaussin M, de Bernard S, Ruffion A, Samarut J, Vlaeminck-Guillem V. Differentially expressed androgen-regulated genes in androgen-sensitive tissues reveal potential biomarkers of early prostate cancer. PLoS One. 2013;8:e66278.

8. Merlo GR, Zerega B, Paleari L, Trombino S, Mantero S, Levi G. Multiple functions of Dlx genes. Genes Dev. 2000:44:619-26.

9. Hamid ARAH, Hoogland AM, Smit F, Jannink S, van Rijt-van de Westerlo C, Jansen CFJ, van Leenders GJLH, Verhaegh GW, Schalken JA. The role of HOXC6 in prostate cancer development. Prostate. 2015;75:1868-76.

10. Van Neste L, Hendriks RJ, Dijkstra S, Trooskens G, Cornel EB, Jannink SA, de Jong $H$, Hessels D, Smit FP, Melchers WJG, de Reijke TM, Vergunst H, Kil P, Knipscheer BC, de Kaa CAH-V, Mulders PFA, van Oort IM, Van Criekinge W, Schalken JA: Detection of High-grade Prostate Cancer Using a Urinary Molecular Biomarker-Based Risk Score. European Urology 2016;70:740-748.

11. Espy MJ, Uhl JR, Sloan LM, Buckwalter SP, Jones MF, Vetter EA, Yao JDC, Wengenack NL, Rosenblatt JE, Cockerill FR, Smith TF. Real-time PCR in clinical microbiology: applications for routine laboratory testing. Clin Microbiol Rev. 2006;19:165-256.

12. Mattocks CJ, Morris MA, Matthijs G, Swinnen E, Corveleyn A, Dequeker E, Müller CR, Pratt V, Wallace A. A standardized framework for the validation and verification of clinical molecular genetic tests. Eur J Hum Genet. 2010; 18:1276-88.

13. Schmittgen TD, Livak KJ. Analyzing real-time PCR data by the comparative C(T) method. Nat Protoc. 2008:3:1101-8.

14. Krouwer JS, Schlain B. A method to quantify deviations from assay linearity. Clin Chem. 1993:39:1689-93.

15. Tholen DW, Kroll MH, Astles JR, Caffo AL, Happe TM, Krouwer JS, Lasky F. EP06-A: Evaluation of the Linearity of Quantitative Measurement Procedures: A Statistical Approach; Approved Guideline. Clin Lab Standards Inst. 2003;23:1.

16. Westgard JO, Groth T, Aronsson T, Falk H, de Verdier CH. Performance characteristics of rules for internal quality control: probabilities for false rejection and error detection. Clin Chem. 1977;23:1857-67.

17. DeLong ER, DeLong DM, Clarke-Pearson DL. Comparing the areas under two or more correlated receiver operating characteristic curves: a nonparametric approach. Biometrics. 1988:44:837-45.

18. Al-Soud WA, Rådström P. Purification and characterization of PCR-inhibitory components in blood cells. J Clin Microbiol. 2001;39:485-93.

19. Sarma PR: Red Cell Indices In: Walker HK, Hall WD, Hurst JW, editors. Clinical Methods: The History, Physical, and Laboratory Examinations. 3rd edition. Boston: Butterworths; 1990. Chapter 152.

20. Bruyninckx R, Buntinx F, Aertgeerts B, Van Casteren V. The diagnostic value of macroscopic haematuria for the diagnosis of urological cancer in general practice. Fam Pract. 2003;53:31-5.

\section{Submit your next manuscript to BioMed Central and we will help you at every step:}

- We accept pre-submission inquiries

- Our selector tool helps you to find the most relevant journal

- We provide round the clock customer support

- Convenient online submission

- Thorough peer review

- Inclusion in PubMed and all major indexing services

- Maximum visibility for your research

Submit your manuscript at www.biomedcentral.com/submit
) Biomed Central 